Research Article

\title{
Boundedness of Solutions for a Class of Second-Order Periodic Systems
}

\author{
Shunjun Jiang ${ }^{1}$ and Yan Ding ${ }^{2}$ \\ ${ }^{1}$ College of Sciences, Nanjing University of Technology, Nanjing 210009, China \\ ${ }^{2}$ Overseas Education College, Nanjing University of Technology, Nanjing 210009, China \\ Correspondence should be addressed to Shunjun Jiang; jiangshunjun@yahoo.com.cn
}

Received 26 June 2013; Accepted 4 November 2013

Academic Editor: Yuriy Rogovchenko

Copyright (c) 2013 S. Jiang and Y. Ding. This is an open access article distributed under the Creative Commons Attribution License, which permits unrestricted use, distribution, and reproduction in any medium, provided the original work is properly cited.

In this paper we study the following second-order periodic system: $x^{\prime \prime}+V^{\prime}(x)+p(x, t)=0$, where $V(x)$ has a singularity. Under some assumptions on the $V(x)$ and $p(x, t)$ by Ortega' small twist theorem, we obtain the existence of quasi-periodic solutions and boundedness of all the solutions.

\section{Introduction and Main Result}

In 1991, Levi [1] considered the following equation:

$$
x^{\prime \prime}+V^{\prime}(x, t)=0
$$

where $V(x, t)$ satisfies some growth conditions and $V(x, t)=$ $V(x, t+1)$. The author reduced the system to a normal form and then applied Moser twist theorem to prove the existence of quasi-periodic solution and the boundedness of all solutions. This result relies on the fact that the nonlinearity $V(x, t)$ can guarantee the twist condition of KAM theorem. Later, several authors improved Levi's result; we refer to [2-4] and the references therein.

Recently, Capietto et al. [5] studied the following equation:

$$
x^{\prime \prime}+V^{\prime}(x)=F(x, t)
$$

wher $F(x, t)=p(t)$ is a $\pi$-periodic function and $V(x)=$ $(1 / 2) x_{+}^{2}+\left(1 /\left(1-x_{-}^{2}\right)^{\nu}\right)-1$, where $x_{+}=\max \{x, 0\}, x_{-}=$ $\max \{-x, 0\}$, and $v$ is a positive integer. Under the LazerLeach assumption that

$$
1+\frac{1}{2} \int_{0}^{\pi} p\left(t_{0}+\theta\right) \sin \theta d \theta>0, \quad \forall t_{0} \in R,
$$

they prove the boundedness of solutions and the existence of quasi-periodic solution by Moser twist theorem. It is the first time that the equation of the boundedness of all solutions is treated in case of a singular potential.

We observe that $F(x, t)=p(t)$ in (2) is smooth and bounded, so a natural question is to find sufficient conditions on $F(x, t)$ such that all solutions of (2) are bounded when $F(x, t)$ is unbounded. The purpose of this paper is to deal with this problem.

Motivated by the papers $[1,5,6]$, we consider the following equation:

$$
x^{\prime \prime}+V^{\prime}(x)+p(x, t)=0
$$

where

$$
V=\frac{1}{2} x_{+}^{2}+\frac{1}{1-x_{-}^{2}}-1, \quad x>-1 .
$$

In order to state our main results, we give some notation and assumptions. Let $\alpha \in(0,1)$ be some fixed constant. Let

$$
\bar{p}=\frac{p(x, t)}{|x|^{\alpha}}, \quad P(x, t)=\int_{0}^{x} p(s, t) d s .
$$

(A1) Assume $p(x, t) \in C^{7,6}\left(S^{1} \times R\right)$ and $\lim _{x \rightarrow+\infty} \bar{p}(x, t)=$ $\bar{p}_{+}(t)$ uniformly in $t$.

(A2) $\lim _{x \rightarrow+\infty} x^{m}\left(\partial^{m+n} \bar{p}(x, t) / \partial x^{m} \partial t^{n}\right)=\bar{p}_{+, m, n}(t)$ uniformly in $t$ for $(m, n)=(6,0),(6,7),(0,7)$, where $\bar{p}_{+, m, 0}(t) \equiv 0$ and $\bar{p}_{+, 6,7}(t) \equiv 0$. 
(A3) We suppose Lazer-Leach assumption holds:

$$
\int_{0}^{\pi} \bar{p}_{+}\left(t_{0}+\theta\right)(\sin \theta)^{1+\alpha} d \theta>0, \quad \forall t_{0} \in R
$$

Our main result is the following theorem.

Theorem 1. Under the assumptions (A1)-(A3), all the solutions of (4) are defined for all $t \in(-\infty,+\infty)$, and for each solution $x(t)$, one has $\sup _{t \in R}\left(|x(t)|+\left|x^{\prime}(t)\right|\right)<+\infty$.

The main idea of our proof is acquired from [6]. The proof of Theorem 1 is based on a small twist theorem due to Ortega [7]. The hypotheses (A1)-(A3) of our theorem are used to prove that the Poincaré mapping of (4) satisfies the assumptions of Ortega's theorem.

Moreover, we have the following theorem on solutions of Mather type.

Theorem 2. Assume that $p(t) \in C$ satisfies (7); then, there is an $\epsilon_{0}>0$ such that, for any $\omega \in\left(1 / \pi, 1 /\left(\pi+\epsilon_{0}\right)\right)$, (4) has a solution $\left(x_{\omega}(t), x_{\omega}^{\prime}(t)\right)$ of Mather type with rotation number $\omega$. More precisely,

Case $1\left(\omega=p / q\right.$ is rational). The solutions $\left(x_{\omega}(t+2 i \pi), x_{\omega}^{\prime}(t+\right.$ $2 i \pi)), 1 \leq i \leq q-1$, are mutually unlinked periodic solution of periodic $q \pi$; moreover, in this case,

$$
\lim _{q \rightarrow \infty} \min _{t \in R}\left|x_{\omega}(t)\right|+\left|x_{\omega}^{\prime}(t)\right|=+\infty .
$$

Case 2 ( $\omega$ is irrational). The solution $\left(x_{\omega}(t), x_{\omega}^{\prime}(t)\right)$ is either a usual quasi-periodic solution or a generalized one.

\section{Proof of Theorem}

2.1. Action-Angle Variables and Some Estimates. Observe that (4) is equivalent to the following Hamiltonian system:

$$
x^{\prime}=\frac{\partial H}{\partial y}, \quad y^{\prime}=-\frac{\partial H}{\partial x}
$$

with the Hamiltonian function

$$
H(x, y, t)=\frac{1}{2} y^{2}+V(x)+P(x, t) .
$$

In order to introduce action and angle variables, we first consider the auxiliary autonomous equation:

$$
x^{\prime}=y, \quad y^{\prime}=-V^{\prime}(x),
$$

which is an integrable Hamiltonian system with Hamiltonian function

$$
H_{1}(x, y, t)=\frac{1}{2} y^{2}+V(x) .
$$

The closed curves $H_{1}(x, y, t)=h>0$ are just the integral curves of (11).

Denote by $T_{0}(h)$ the time period of the integral curve $\Gamma_{h}$ of (11) defined by $H_{1}(x, y, t)=h$ and by $I$ the area enclosed by the closed curve $\Gamma_{h}$ for every $h>0$. Let $-1<-\alpha_{h}<0<$ $\beta_{h}$ be such that $V\left(-\alpha_{h}\right)=V\left(\beta_{h}\right)=h$. It is easy to see that

$$
I_{0}(h)=2 \int_{-\alpha_{h}}^{\beta_{h}} \sqrt{2(h-V(s))} d s, \quad \forall h>0,
$$

$$
T_{0}(h)=I_{0}^{\prime}(h)=2 \int_{\alpha_{h}}^{\beta_{h}} \frac{1}{\sqrt{2(h-V(s))}} d s, \quad \forall h>0 .
$$

By direct computation we get

$$
\begin{aligned}
I_{0}(h) & =2 \int_{0}^{\beta_{h}} \sqrt{2(h-V(s))} d s+2 \int_{-\alpha_{h}}^{0} \sqrt{2(h-V(s))} d s \\
& =\pi h+2 \int_{0}^{\alpha_{h}} \sqrt{2(h-V(-s))} d s,
\end{aligned}
$$

so

$$
T_{0}(h)=\pi+\int_{0}^{\alpha_{h}} \frac{1}{\sqrt{2(h-V(-s))}} d s .
$$

We then have

$$
I_{0}(h)=I_{-}(h)+I_{+}(h), \quad T_{0}(h)=T_{-}(h)+T_{+}(h),
$$

where

$$
\begin{array}{ll}
I_{-}(h)=2 \int_{0}^{-\alpha_{h}} \sqrt{2(h-V(s))} d s, & I_{+}(h)=\pi h, \\
T_{-}(h)=2 \int_{0}^{-\alpha_{h}} \frac{1}{\sqrt{2(h-V(-s))}} d s, & T_{+}(h)=\pi .
\end{array}
$$

We now give the estimates on the functions $I_{-}$and $T_{-}$.

Lemma 3. One has

$$
\begin{aligned}
& h^{n}\left|\frac{d^{n} T_{-}(h)}{d h^{n}}\right| \leq C h^{-1 / 2}, \\
& h^{n}\left|\frac{d^{n} I_{-}(h)}{d h^{n}}\right| \leq C h^{1 / 2},
\end{aligned}
$$

where $n=0,1, \ldots, 6, h \rightarrow \infty$. Note that here and below one always uses $C, C_{0}$, or $C_{0}^{\prime}$ to indicate some constants.

Proof. Now we estimate the first inequality. We chose $V(s) / h=\eta$ as the new variable of integration; then we have

$$
\begin{aligned}
T_{-}(h) & =\int_{-\alpha_{h}}^{0} \frac{1}{\sqrt{2(h-V(s))}} d s \\
& =\int_{0}^{1} \frac{\sqrt{h}}{V^{\prime}(s(\eta, h))} \frac{1}{\sqrt{2(1-\eta)}} d \eta .
\end{aligned}
$$

Since $V(s)=\left(1 /\left(1-s^{2}\right)\right)-1$ and $V(s) / h=\eta$, we have $s=$ $\sqrt{\eta h /(1+\eta h)}$. By direct computation, we have

$$
V^{\prime}(s)=\frac{2 s}{\left(1-s^{2}\right)^{2}}=\frac{2 \sqrt{\eta h}(1+\eta h)^{2}}{\sqrt{1+\eta h}},
$$


and then we get

$$
\begin{array}{r}
T_{-}^{(n)}(h)=\frac{(-3 / 2) !}{((-3 / 2)-n) !} \int_{0}^{1} \frac{\eta^{n}}{\sqrt{2 \eta(1-\eta)}(1+\eta h)^{(3 / 2)+n}} d \eta \\
n=0,1, \ldots, 6 .
\end{array}
$$

When $0 \leq \eta \leq h^{-1}$ and $h$ is sufficiently large, there exits $C_{0}$ such that $1-\eta>C_{0}$, so we have

$$
\begin{aligned}
& \int_{0}^{h^{-1}} \frac{\eta^{n}}{\sqrt{2 \eta(1-\eta)}(1+\eta h)^{(3 / 2)+n}} d \eta \\
& \quad \leq C \int_{0}^{h^{-1}} \frac{\eta^{n}}{\sqrt{2 \eta(1-\eta)}} d \eta \\
& \quad \leq \frac{C}{C_{0}} \int_{0}^{h^{-1}} \eta^{n-(1 / 2)} d \eta \leq C h^{-(1 / 2)-n} .
\end{aligned}
$$

Since $h^{-2 / 3} \leq \eta \leq 1$, we have

$$
h^{1 / 3}<1+h^{1 / 3} \leq 1+\eta h \leq 1+h,
$$

and then

$$
\begin{aligned}
\int_{h^{-2 / 3}}^{1} & \frac{\eta^{n}}{\sqrt{2 \eta(1-\eta)(1+\eta h)^{(3 / 2)+n}}} d \eta \\
& \leq C \int_{h^{-2 / 3}}^{1} \frac{\eta^{n} h^{n}}{\sqrt{2 \eta(1-\eta)} h^{n}(1+\eta h)^{n}(1+\eta h)^{3 / 2}} d \eta \\
& \leq C \int_{h^{-2 / 3}}^{1} \frac{1}{\sqrt{2 \eta(1-\eta)} h^{n}(1+\eta h)^{3 / 2}} d \eta \\
& \leq C \int_{h^{-2 / 3}}^{1} \frac{1}{\sqrt{2 \eta(1-\eta)} h^{n} h^{1 / 2}} d \eta \\
& \leq C h^{(-1 / 2)-n \int_{0}^{1} \frac{1}{\sqrt{2 \eta(1-\eta)}} d \eta \leq C h^{(-1 / 2)-n} .}
\end{aligned}
$$

Observing that there is $C_{0}>0$ such that $\sqrt{1-\eta} \geq C_{0}$ when $h^{-1} \leq \eta \leq h^{-2 / 3}$ and $h \rightarrow+\infty$, we have

$$
\begin{aligned}
\int_{h^{-1}}^{h^{-2 / 3}} & \frac{\eta^{n}}{\sqrt{2 \eta(1-\eta)}(1+\eta h)^{(3 / 2)+n}} d \eta \\
\leq & C_{1} h^{(-3 / 2)-n} \int_{h^{-1}}^{h^{-2 / 3}} \frac{1}{\sqrt{2 \eta(1-\eta)} \eta^{3 / 2}} d \eta \\
\leq & \frac{C_{1}}{C_{0}} h^{(-3 / 2)-n} \int_{h^{-1}}^{h^{-2 / 3}} \frac{1}{\eta^{2}} d \eta=\left.\frac{C_{1}}{C_{0}} h^{(-3 / 2)-n} \frac{1}{\eta}\right|_{h_{-1}} ^{h^{-2 / 3}} \\
\quad & \frac{C_{1}}{C_{0}} h^{(-3 / 2)-n}\left(h-h^{2 / 3}\right) \leq C h^{-(1 / 2)-n} .
\end{aligned}
$$

By (22)-(25) we have $T_{-}^{(n)}(h) \leq C h^{(-1 / 2)-n}, n=0,1, \ldots, 6$.
The proof of the second inequality is similar to the first one, so we only give a brief proof.

We choose $V(s) / h=\eta$ as the new variable of integration, so we have

$$
\begin{gathered}
\frac{\partial s}{\partial h}=\frac{\eta}{V^{\prime}}, \quad s=\sqrt{\frac{\eta h}{1+\eta h}}, \\
V^{\prime}(s)=\frac{2 s}{\left(1-s^{2}\right)^{2}}=\frac{2 \sqrt{\eta h}(1+\eta h)^{2}}{\sqrt{1+\eta h}} .
\end{gathered}
$$

By direct computation, we have

$$
I_{-}(h)=2 \int_{-\alpha_{h}}^{0} \sqrt{2(h-V(s))} d s=h \int_{0}^{1} \frac{\sqrt{2(1-\eta)}}{\sqrt{\eta}(1+\eta h)^{3 / 2}} d \eta .
$$

By (27), we can easily get

$$
\begin{aligned}
I_{-}^{(n)}(h)= & I_{-1}^{(n)}(h)+I_{-2}^{(n)}(h) \\
= & n \frac{(-3 / 2) !}{((-3 / 2)-n+1) !} \\
& \times \int_{0}^{1} \frac{\sqrt{2(1-\eta)}}{\sqrt{\eta}} \frac{\eta^{n-1}}{(1+\eta h)^{(3 / 2)+n-1}} d \eta \\
& +\frac{(-3 / 2) !}{((-3 / 2)-n) !} h \int_{0}^{1} \frac{\sqrt{2(1-\eta)}}{\sqrt{\eta}} \frac{\eta^{n}}{(1+\eta h)^{(3 / 2)+n}} d \eta
\end{aligned}
$$

where $n=0,1, \ldots, 6$.

By a similar way to that used in estimating $T_{-}^{(n)}(h)$, we get

$$
I_{-1}^{(n)}(h) \leq C h^{(1 / 2)-n}, \quad I_{-2}^{(n)}(h) \leq C h^{(1 / 2)-n},
$$

which means that

$$
I_{-}^{(n)}(h) \leq C h^{(1 / 2)-n}, \quad n=0,1, \ldots, 6 .
$$

Thus Lemma 3 is proved.

Remark 4. It follows from the definitions of $T_{+}(h), T_{-}(h)$ and Lemma 3 that

$$
\lim _{h \rightarrow+\infty} T_{-}(h)=0, \quad \lim _{h \rightarrow+\infty} T_{+}(h)=\pi .
$$

Thus the time period $T_{0}(h)$ is dominated by $T_{+}(h)$ when $h$ is sufficiently large. From the relation between $T_{-}(h)$ and $I_{-}(h)$, we know $I_{0}(h)$ is dominated by $I_{+}(h)$ when $h$ is sufficiently large.

Remark 5. It also follows from the definition of $I(h), I_{-}(h)$, $I_{+}(h)$ and Remark 4 that

$$
\left|h^{n} \frac{d^{n} I_{0}(h)}{d h^{n}}\right| \leq C_{0} I_{0}(h), \quad \text { for } n \geq 1 .
$$


Remark 6. Note that $h=h_{0}\left(I_{0}\right)$ is the inverse function of $I_{0}$. By Remark 5, we have

$$
\left|I^{n} \frac{d^{n} h(I)}{d I^{n}}\right| \leq C_{0} h(I) \quad \text { for } n \geq 1 .
$$

We now carry out the standard reduction to the actionangle variables. For this purpose, we define the generating function $S(x, I)=\int_{C} \sqrt{2(h-V(s))} d s$, where $C$ is the part of the closed curve $\Gamma_{h}$ connecting the point on the $y$-axis and point $(x, y)$.

We define the well-known map $(\theta, I) \rightarrow(x, y)$ by

$$
y=\frac{\partial S}{\partial x}(x, I), \quad \theta=\frac{\partial S}{\partial I}(x, I),
$$

which is symplectic since

$$
\begin{aligned}
& d x \wedge d y=d x \wedge\left(S_{x x} d x+S_{x I} d I\right)=S_{x I} d x \wedge d I, \\
& d \theta \wedge d I=\left(S_{I x} d x+S_{I I} d I\right) \wedge d I=S_{I x} d \wedge d I .
\end{aligned}
$$

From the above discussion, we can easily get

$$
\begin{aligned}
& \left\{\begin{array}{l}
\frac{\pi}{T_{0}(h(x, y))} \\
\times\left(\frac{T_{-}(h(x, y))}{2}+\arcsin \frac{x}{\sqrt{2(h(x, y))}}\right),
\end{array}\right. \\
& \frac{\pi}{T_{0}(h(x, y))} \\
& \times\left(\frac{T_{-}(h(x, y))}{2}+\pi+\arcsin \frac{x}{\sqrt{2(h(x, y))}}\right), \\
& \theta= \begin{cases}\pi & \text { if } x>0, y<0, \\
\frac{\pi(h, y))}{T_{0}(h(x)} & \end{cases} \\
& \overline{T_{0}(h(x, y))} \\
& \times\left(\int_{-\alpha_{h}}^{x} \frac{1}{\sqrt{2\left(h(x, y)+1-\left(1-s^{2}\right)^{-1}\right)}} d s\right), \\
& \text { if } x<0, y>0 \text {, } \\
& \frac{\pi}{T_{0}(h(x, y))} \\
& \times\left(T_{0}(h(x, y))-\int_{-\alpha_{h}}^{x} \frac{1}{\sqrt{2\left(h(x, y)+1-\left(1-s^{2}\right)^{-1}\right)}} d s\right), \\
& \text { if } x<0, y<0 \text {, } \\
& I(x, y)=I_{0}(h(x, y))=2 \int_{-\alpha_{h}}^{\beta_{h}} \sqrt{2(h(x, y)-V(s))} d s .
\end{aligned}
$$

In the new variables $(\theta, I)$, the system (9) becomes

$$
\theta^{\prime}=\frac{\partial H}{\partial I}, \quad I^{\prime}=-\frac{\partial H}{\partial \theta}
$$

where

$$
H(\theta, I, t)=\pi h_{0}(I)+\pi P(x(I, \theta), t) .
$$

In order to estimate $\pi P(I, \theta)$, we need the estimate on the function $x(I, \theta)$.

Lemma 7. For I sufficiently large and $-\alpha_{h} \leq x<0$, the following estimates hold:

$$
\left|I^{n} \frac{\partial^{n} x(I, \theta)}{\partial I^{n}}\right| \leq c \sqrt{I}, \quad \text { for } 0 \leq n \leq 6
$$

The Lemma was first proved in [1], and later [5] gives a different proof; [8] using the method of induction hypothesis also gives another proof. So, for concision, we omit the proof.

2.2. New Action and Angle Variables. Now we are concerned with the Hamiltonian system (37) with Hamiltonian function $H(\theta, I, t)$ given by $(38)$. Note that

$$
I d \theta-H d t=-(H d t-I d \theta) .
$$

This means that if one can solve $I$ from (38) as a function of $H$ (using $\theta$ and $t$ as parameters), then

$$
\frac{d H}{d \theta}=-\frac{\partial I}{\partial t}(t, H, \theta), \quad \frac{d t}{d \theta}=\frac{\partial I}{\partial H}(t, H, \theta)
$$

is also a Hamiltonian system with Hamiltonian function $I$ and now the action, angle, and time variables are $H, t$, and $\theta$.

From (38) and Lemma 3, we have

$$
\frac{\partial H}{\partial I} \longrightarrow 1, \quad \text { as } I \longrightarrow+\infty
$$

So we assume that $I$ can be written as

$$
I=I_{0}\left(\frac{H}{\pi}+R(H, t, \theta)\right)
$$

where $R$ satisfies $|R|<H / \pi$. Recalling that $h_{0}$ is the inverse function of $I_{0}$, we have

$$
\frac{H}{\pi}+R(H, t, \theta)=h_{0}(I)
$$

which implies that

$$
R(H, t, \theta)=P(x(I, \theta), t) .
$$

As a consequence, $R$ is implicitly defined by

$$
R(H, t, \theta)=P\left(x\left(I_{0}\left(\frac{H}{\pi}+R(H, t, \theta)\right), \theta\right), t\right) .
$$

For the estimates of $R$, we need the following lemmas.

Lemma 8. Let $f(x, t)$ and $g(x, t)$ be continuously differentiable for $(x, t) \in[0,+\infty) \times I$, where $I$ is an interval of $R$. If

(1) $g(x, t) \rightarrow \infty$ as $x \rightarrow+\infty$, uniformly with respect to $t \in I$, 
(2) $f_{x}^{\prime}(x, t) / g_{x}^{\prime}(x, t) \rightarrow h(t)$ as $x \rightarrow+\infty$, uniformly with respect to $t \in I$,

then one has $f(x, t) / g(x, t) \rightarrow h(t)$ as $x \rightarrow+\infty$, uniformly with respect to $t \in I$.

Proof. For any $0<\epsilon<1$, there exits $X_{1}$, such that if $x>X_{1}$, we have

$$
\left|\frac{f_{x}^{\prime}(x, t)}{g_{x}^{\prime}(x, t)}-h(t)\right| \leq \frac{\epsilon}{2}, \quad \forall t \in I .
$$

Let $x_{0}=X_{1}+1$. Then by Lagrangian differential mean value theorem, it follows that, for all $x>X_{1}$, we have

$$
\left|\frac{f(x, t)-f\left(x_{0}, t\right)}{g(x, t)-g\left(x_{0}, t\right)}-h(t)\right| \leq \frac{\epsilon}{2}, \quad \forall t \in I .
$$

Moreover, there exists a constant $M>0$ such that

$$
\left|g\left(x_{0}, t\right) \frac{f(x, t)-f\left(x_{0}, t\right)}{g(x, t)-g\left(x_{0}, t\right)}-f\left(x_{0}, t\right)\right| \leq M, \quad \forall t \in I .
$$

By condition (A1), there exists $X>X_{1}$; we have $|g(x, t)|>$ $2 M / \epsilon$.

$$
\begin{aligned}
& \text { Thus } \\
& \left|\frac{f(x, t)}{g(x, t)}-h(t)\right| \leq\left|\frac{f(x, t)-f\left(x_{0}, t\right)}{g(x, t)-g\left(x_{0}, t\right)}-h(t)\right| \\
& \quad+\left|\frac{1}{g(x, t)}\left(g\left(x_{0}, t\right) \frac{f(x, t)-f\left(x_{0}, t\right)}{g(x, t)-g\left(x_{0}, t\right)}-f\left(x_{0}, t\right)\right)\right|<\epsilon .
\end{aligned}
$$

Lemma 9. Under the assumptions (A1) and (A2), the following results hold:

(1) $\lim _{x \rightarrow+\infty}\left(x^{m}\left(\partial^{m+n} \bar{p}(x, t) / \partial x^{m} \partial t^{n}\right)\right)=\bar{p}_{+, m, n}(t)$ uniformly in $t$, for $0 \leq m \leq 6$ and $0 \leq n \leq 7$, where $\bar{p}_{+, m, n}(t)=0$ for $1 \leq m \leq 6,0 \leq n \leq 7$ and $\bar{p}_{+, 0, n}(t)=\bar{p}^{(n)}(t)$ for $0 \leq n \leq 7$.

(2) $\lim _{x \rightarrow+\infty}\left(x^{m}\left(\partial^{m+n} / \partial x^{m} \partial t^{n}\right)\left(P(x, t) /|x|^{\sigma} x\right)\right)=(1 /$ $(\sigma+1)) \bar{p}_{+, m, n}(t)$, for $0 \leq m \leq 6$ and $0 \leq n \leq 7$, where $\bar{p}_{+, 7, n}(t)=0$ for $0 \leq n \leq 7$.

Proof. Result (1) is similar to Lemma 2.1 in [9], so we omit the proof.

For result (2), we first prove that, for $1 \leq m \leq 6$ and $0 \leq$ $n \leq 7$,

$$
\begin{array}{r}
\lim _{x \rightarrow+\infty} x^{m} \frac{\left(\partial^{m+n} / \partial x^{m} \partial t^{n}\right) p(t, x)}{|x|^{\sigma}} \\
=\sigma \cdots(\sigma-m+1) \bar{p}_{+}^{(n)}(t) .
\end{array}
$$

For $m=1$ and $0 \leq n \leq 7$, by result (1), we have that

$$
\lim _{x \rightarrow+\infty} x\left\{-\sigma \cdot \frac{\left(\partial^{n} / \partial t^{n}\right) p(t, x)}{|x|^{\sigma} \cdot x}+\frac{\left(\partial^{1+n} / \partial x \partial t^{n}\right) p(t, x)}{|x|^{\sigma}}\right\}=0,
$$

uniformly in $t$. It follows that

$$
\lim _{x \rightarrow+\infty} x \cdot \frac{\left(\partial^{1+n} / \partial x \partial t^{n}\right) p(t, x)}{|x|^{\sigma}}=\sigma \cdot \bar{p}_{+}^{(n)}(t) .
$$

This means that (51) holds for $m=1$. For $m=2$, combining this with result (1) and the result of (51) for $m=1$, we have that (51) still holds. Inductively we can prove that (51) holds for all $1 \leq m \leq 6$.

Obviously, it follows that

$$
\begin{aligned}
& \frac{\partial^{n}}{\partial t^{n}} \frac{P(t, x)}{|x|^{\sigma} \cdot x}-\frac{1}{1+\alpha} \cdot \bar{p}_{+}^{(n)}(t) \\
& \quad=\frac{\int_{0}^{x}\left[\left(\partial^{n} / \partial t^{n}\right) p(t, x)-\bar{p}_{+}^{(n)}(t)|s|^{\sigma}\right] d s}{|x|^{\sigma} \cdot x} .
\end{aligned}
$$

Using Lemma 8 and the first result (1) for $m=0$, it follows that

$$
\lim _{x \rightarrow+\infty} \frac{\partial^{n}}{\partial t^{n}} \frac{P(t, x)}{|x|^{\sigma} \cdot x}=\frac{1}{\sigma+1} \bar{p}_{+}^{(n)}(t),
$$

for $0 \leq n \leq 7$. In a similar way to the proof of (51), we have

$$
\begin{aligned}
& \lim _{x \rightarrow+\infty} x^{m} \frac{\partial^{m+n}}{\partial x^{m} \partial t^{n}} \frac{P(x, t)}{|x|^{\sigma} x} \\
& =\frac{1}{\sigma+1} \bar{p}_{+, m, n}(t), \quad \text { for } 0 \leq m \leq 6,0 \leq n \leq 7,
\end{aligned}
$$

where $\bar{p}_{+, 7, n}(t)=0$. Thus we proved Lemma 9 .

Now we give the estimates of $R$. By Lemma 9, in a similar way to that for Lemma 2.3 in [5], we have the following lemma.

Lemma 10. The function $R(H, t, \theta)$ satisfies the following estimates:

$$
\left|\frac{\partial^{m+l} R(H, t, \theta)}{\partial H^{m} \partial t^{l}}\right| \leq H^{(\alpha+1) / 2}, \quad \text { for } m+l \leq 6 .
$$

Moreover, by the implicit function theorem, there exists a function $R_{1}=R_{1}(t, H, \theta)$ such that

$$
R(H, t, \theta)=P(x(H, \theta), t)+R_{1}(H, t, \theta) .
$$

Since

$$
\begin{aligned}
R_{1}(H, t, \theta)= & R(H, t, \theta)-P(x(H, \theta), t) \\
= & P\left\{x\left[I_{0}\left(\frac{H}{\pi}+R(H, t, \theta)\right), \theta\right], t\right\} \\
& -P(x(H, \theta), t) \\
= & \int_{0}^{1} p\left\{x\left[H+s\left(\pi R+I_{-}\right), \theta\right], t\right\} \\
& \cdot \frac{\partial x}{\partial I}\left(H+s\left(\pi R+I_{-}\right), \theta\right) \cdot\left(\pi R+I_{-}\right) d s,
\end{aligned}
$$

by Lemmas 3 and 10, we have the estimates on $R_{1}(H, t, \theta)$. 
Lemma 11. Consider the following:

$$
\left|\frac{\partial^{k+l} R_{1}(H, t, \theta)}{\partial^{k} H \partial^{l} t}\right|<H^{\alpha / 2} \quad \text { for } k+l \leq 6 .
$$

For the estimate of $I((H / \pi)+R)$, we need the estimate on $I_{-}((H / \pi)+R)$. By Lemma 3 and noticing that $|R|<H / \pi$, we have the following lemma.

Lemma 12. Consider the following:

$$
\left|\frac{\partial^{k+l} I_{-}((H / \pi)+R)}{\partial^{k} H \partial^{l} t}\right|<H^{1 / 2} \quad \text { for } k+l \leq 6 .
$$

Now the new Hamiltonian function $I=I(t, H, \theta)$ is written in the form

$$
\begin{aligned}
I & =I_{0}\left(\frac{H}{\pi}+R\right)=I_{+}\left(\frac{H}{\pi}+R\right)+I_{-}\left(\frac{H}{\pi}+R\right) \\
& =H+\pi R(H, t, \theta)+I_{-}\left(\frac{H}{\pi}+R\right) \\
& =H+\pi P(x(H, \theta), t)+R_{1}(H, t, \theta)+I_{-}\left(\frac{H}{\pi}+R\right) .
\end{aligned}
$$

The system (41) is of the form

$$
\begin{aligned}
\frac{d t}{d \theta}=\frac{\partial I}{\partial H}= & 1+\pi \frac{\partial x}{\partial H}(H, \theta) p(x(H, \theta), t) \\
& +\frac{\partial R_{1}}{\partial H}(H, t, \theta)+\frac{\partial I_{-}}{\partial H}(H, t, \theta), \\
\frac{d H}{d \theta}=-\frac{\partial I}{\partial t}= & -\pi \frac{\partial P}{\partial t}(x(\theta, H), t) \\
& -\frac{\partial R_{1}}{\partial t}(t, H, \theta)-\frac{\partial I_{-}}{\partial t}(H, t, \theta) .
\end{aligned}
$$

Introduce a new action variable $\rho \in[1,2]$ and a parameter $\epsilon>0$ by $H=\epsilon^{-2} \rho$. Then, $H \gg 1 \Leftrightarrow 0<\epsilon \ll 1$. Under this transformation, the system (63) is changed into the form

$$
\begin{aligned}
\frac{d t}{d \theta}=\frac{\partial I}{\partial H}= & 1+\pi \frac{\partial x}{\partial H}(H, \theta) p(x(H, \theta), t) \\
& +\frac{\partial R_{1}}{\partial H}(H, t, \theta)+\frac{\partial I_{-}}{\partial H}(H, t, \theta), \\
\frac{d \rho}{d \theta}=-\frac{\partial I}{\partial t}= & -\epsilon^{2}\left[\pi \frac{\partial P}{\partial t}(x(\theta, H), t)\right. \\
& \left.+\frac{\partial R_{1}}{\partial t}(H, t, \theta)+\frac{\partial I_{-}}{\partial t}(H, t, \theta)\right],
\end{aligned}
$$

which is also Hamiltonian system with the new Hamiltonian function

$$
\begin{aligned}
\Gamma(t, \rho, \theta ; \epsilon)= & \rho+\pi \epsilon^{-2} P\left(x\left(\theta, \epsilon^{-2} \rho\right), t\right) \\
& +\epsilon^{-2} R_{1}\left(\epsilon^{-2} \rho, \theta, t\right)+\epsilon^{-2} I_{-}\left(\epsilon^{-2} \rho, \theta, t\right) .
\end{aligned}
$$

Obviously, if $\epsilon \ll 1$, the solution $\left(t\left(\theta, t_{0}, \rho_{0}\right), \rho\left(\theta, t_{0}, \rho_{0}\right)\right)$ of (64) with the initial date $\left(t_{0}, \rho_{0}\right) \in R \times[1,2]$ is defined in the interval $\theta \in[0,2 \pi]$ and $\rho\left(\theta, t_{0}, \rho_{0}\right) \in[(1 / 2), 3]$. So the Poincare map of (64) is well defined in the domain $R \times[1,2]$.
Lemma 13 (see [6] Lemma 5.1). The Poincaré map of (64) has intersection property.

The proof is similar to the corresponding one in [6].

For convenience we introduce the notation $O_{k}(1)$ and $o_{k}(1)$. We say a function $f(t, \rho, \theta, \epsilon) \in O_{k}(1)$ if $f$ is smooth in $(t, \rho)$ and, for $k_{1}+k_{2} \leq k$,

$$
\left|\frac{\partial^{k_{1}+k_{2}}}{\partial t^{k_{1}} \partial \rho^{k_{2}}} f(t, \rho, \theta, \epsilon)\right| \leq C,
$$

for some constant $C>0$ which is independent of the arguments $t, \rho, \theta$, and $\epsilon$.

Similarly, we say $f(t, \rho, \theta, \epsilon) \in o_{k}(1)$ if $f$ is smooth in $(t, \rho)$ and, for $k_{1}+k_{2} \leq k$,

$$
\lim _{\epsilon \rightarrow 0}\left|\frac{\partial^{k_{1}+k_{2}}}{\partial t^{k_{1}} \partial \rho^{k_{2}}} f(t, \rho, \theta, \epsilon)\right|=0
$$

uniformly in $(t, \rho, \theta)$.

2.3. Poincaré Map and Twist Theorems. We will use Ortega' small twist theorem to prove that the Pioncaré map $P$ has an invariant closed curve, if $\epsilon$ is sufficiently small. Let us first recall the theorem in [7].

Lemma 14 (Ortega's Theorem). Let $A=\mathbb{S}^{1} \times[a, b]$ be a finite cylinder with universal cover $\mathbb{A}=\mathbb{R} \times[a, b]$. The coordinate in $\mathbb{A}$ is denoted by $(\tau, \nu)$. Consider a map

$$
\bar{f}: A \longrightarrow \mathbb{S} \times \mathbb{R} .
$$

One assumes that the map has the intersection property. Suppose that $f: A \rightarrow \mathbb{R} \times \mathbb{R},\left(\tau_{0}, v_{0}\right) \rightarrow\left(\tau_{1}, v_{1}\right)$ is a lift of $\bar{f}$ and it has the form

$$
\begin{gathered}
\tau_{1}=\tau_{0}+2 N \pi+\delta l_{1}\left(\tau_{0}, v_{0}\right)+\delta \tilde{g}_{1}\left(\tau_{0}, v_{0}\right), \\
v_{1}=v_{0}+\delta l_{2}\left(\tau_{0}, v_{0}\right)+\delta \tilde{g}_{2}\left(\tau_{0}, v_{0}\right),
\end{gathered}
$$

where $N$ is an integer and $\delta \in(0,1)$ is a parameter. The functions $l_{1}, l_{2}, \tilde{g}_{1}$, and $\tilde{g}_{2}$ satisfy

$$
\begin{array}{r}
l_{1} \in C^{6}(A), \quad l_{1}\left(\tau_{0}, v_{0}\right)>0, \quad \frac{\partial l_{1}}{\partial \nu_{0}}\left(\tau_{0}, v_{0}\right)>0, \\
\forall\left(\tau_{0}, v_{0}\right) \in A, \\
l_{2}(\cdot, \cdot), \widetilde{g}_{1}(\cdot, \cdot, \epsilon), \widetilde{g}_{2}(\cdot, \cdot, \epsilon) \in C^{5}(A) .
\end{array}
$$

In addition, one assumes that there is a function $I: A \rightarrow$ $R$ satisfying

$$
\begin{aligned}
& I \in C^{6}(A), \quad \frac{\partial I}{\partial \nu_{0}}\left(\tau_{0}, \nu_{0}\right)>0, \quad \forall\left(\tau_{0}, v_{0}\right) \in A, \\
& l_{1}\left(\tau_{0}, v_{0}\right) \cdot \frac{\partial I}{\partial \tau_{o}}\left(\tau_{0}, v_{0}\right)+l_{2}\left(\tau_{0}, v_{0}\right) \cdot \frac{\partial I}{\partial \nu_{0}}\left(\tau_{0}, v_{0}\right)=0, \\
& \forall\left(\tau_{0}, v_{0}\right) \in A \text {. }
\end{aligned}
$$


Moreover, suppose that there are two numbers $\tilde{a}$ and $\tilde{b}$ such that $a<\widetilde{a}<\widetilde{b}<b$ and

$$
I_{M}(a)<I_{m}(\widetilde{a}) \leq I_{M}(\widetilde{a})<I_{m}(\widetilde{b}) \leq I_{M}(\widetilde{b})<I_{m}(b),
$$

where

$$
I_{M}(r)=\max _{\rho \in S^{1}} I\left(\rho_{o}, \tau_{o}\right), \quad I_{m}(r)=\min _{\rho \in S^{1}} I\left(\rho_{o}, \tau_{o}\right) .
$$

Then there exist $\epsilon>0$ and $\Delta>0$ such that, if $\delta<\Delta$ and

$$
\left\|\widetilde{\mathfrak{g}}_{1}(\cdot, \cdot, \epsilon)\right\|_{C^{5}(A)}+\left\|\widetilde{\mathfrak{g}}_{2}(\cdot, \cdot, \epsilon)\right\|_{C^{5}(A)}<\epsilon,
$$

the mapping $\bar{f}$ has an invariant curve in $\Gamma_{A}$. The constant $\epsilon$ is independent of $\delta$.

We make the ansatz that the solution of (64) with the initial condition $(t(0), \rho(0))=\left(t_{0}, \rho_{0}\right)$ is of the form

$$
\begin{aligned}
& t=t_{0}+\theta+\epsilon^{1-\alpha} \Sigma_{1}\left(t_{0}, \rho_{0}, \theta ; \epsilon\right), \\
& \rho=\rho_{0}+\epsilon^{1-\alpha} \Sigma_{2}\left(t_{0}, \rho_{0}, \theta ; \epsilon\right) .
\end{aligned}
$$

Then, the Poincaré map of (64) is

$$
\begin{aligned}
& P: t_{1}=t_{0}+2 \pi+\epsilon^{1-\alpha} \Sigma_{1}\left(t_{0}, \rho_{0}, 2 \pi ; \epsilon\right), \\
& \rho_{1}=\rho_{0}+\epsilon^{1-\alpha} \Sigma_{2}\left(t_{0}, \rho_{0}, 2 \pi ; \epsilon\right) .
\end{aligned}
$$

The functions $\Sigma_{1}$ and $\Sigma_{2}$ satisfy

$$
\begin{aligned}
\Sigma_{1}= & \pi \epsilon^{\alpha-1} \int_{0}^{\theta} \frac{\partial x}{\partial H}\left(\epsilon^{-2} \rho, \theta\right) p\left(x\left(\epsilon^{-2} \rho, \theta\right), t\right) d \theta \\
& +\epsilon^{\alpha-1} \int_{0}^{\theta}\left(\frac{\partial R_{1}}{\partial H}\left(\epsilon^{-2} \rho, t, \theta\right)+\frac{\partial I_{-}}{\partial H}\left(\epsilon^{-2} \rho, t, \theta\right)\right) d \theta, \\
\Sigma_{2}= & -\pi \epsilon^{\alpha+1} \int_{0}^{\theta} \frac{\partial P}{\partial t}\left(x\left(\epsilon^{-2} \rho, \theta\right), t\right) d \theta \\
& -\epsilon^{\alpha+1} \int_{0}^{\theta}\left(\frac{\partial R_{1}}{\partial t}\left(\epsilon^{-2} \rho, t, \theta\right)-\frac{\partial I_{-}}{\partial t}\left(\epsilon^{-2} \rho, t, \theta\right)\right) d \theta,
\end{aligned}
$$

where $t=t_{0}+\theta+\epsilon^{1-\alpha} \Sigma_{1}$ and $\rho=\rho_{0}+\epsilon^{1-\alpha} \Sigma_{2}$. By Lemmas 9,11 , and 12 , we know that

$$
\left|\Sigma_{1}\right|+\left|\Sigma_{2}\right| \leq C \quad \text { for } \theta \in[0,2 \pi] .
$$

Hence, for $\rho_{0} \in[1,2]$, we may choose $\epsilon$ sufficiently small such that

$$
\rho_{0}+\epsilon \Sigma_{2} \geq \frac{\rho_{0}}{2} \geq \frac{1}{2}
$$

Moreover we can prove that

$$
\Sigma_{1}, \Sigma_{2} \in O_{6}(1) .
$$

In a similar way to that used for estimating $R_{1}$, by direct calculation we have the following lemma.
Lemma 15. The following estimates hold:

$$
\begin{aligned}
& P\left(x\left(\epsilon^{-2} \rho, \theta\right), t\right)-P\left(x\left(\epsilon^{-2} \rho_{0}, \theta\right), t_{0}\right) \in \epsilon^{-\alpha} O_{6}(1), \\
& \frac{\partial P}{\partial t}\left(x\left(\epsilon^{-2} \rho, \theta\right), t\right)-\frac{\partial P}{\partial t}\left(x\left(\epsilon^{-2} \rho_{0}, \theta\right), t_{0}\right) \in \epsilon^{2-\alpha} O_{6}(1) .
\end{aligned}
$$

Now we give an asymptotic expression of Poincaré map of (63); that is, we study the behavior of the functions $\Sigma_{1}$ and $\Sigma_{2}$ at $\theta=\pi$ as $\epsilon \rightarrow 0$. In order to estimate $\Sigma_{1}$ and $\Sigma_{2}$, we need to introduce the following definition and lemma. Let

$$
\begin{aligned}
& \Theta_{+}(I)=\text { meas }\left\{\theta \in[0, \pi], x\left(H_{0}, \theta\right)>0\right\}, \\
& \Theta_{-}(I)=T_{0}-\Theta_{+}(I),
\end{aligned}
$$

where $H_{0}=\epsilon^{-2} \rho_{0}$.

Lemma 16. Consider the following:

$$
\Theta_{+}(I)=\pi+\epsilon O_{6}(1), \quad \Theta_{-}(I)=\epsilon O_{6}(1) .
$$

Proof. This Lemma was proved in [5], so we omit the details. For estimate $\Sigma_{1}$ and $\Sigma_{2}$, we need the estimates of $x$ and $x_{H}$.

We recall that, when $x<0$, we have

$$
\left|x\left(H_{0}, \theta\right)\right|=O_{6}(1), \quad\left|x_{H}\left(H_{0}, \theta\right)\right|=\epsilon^{2} O_{5}(1) .
$$

When $x>0$, by the definition of $\theta$, we have

$$
\arcsin \frac{x\left(H_{0}, \theta\right)}{\sqrt{2 h}}=\frac{T_{0}(h)}{\pi} \theta-\frac{T_{-}(h)}{2}=\theta+\epsilon^{2} O_{5}(1),
$$

which yields that

$$
\begin{aligned}
& x\left(H_{0}, \theta\right)=\sqrt{\frac{2 H_{0}}{\pi}} \sin \theta+O_{5}(1), \\
& x_{H}\left(H_{0}, \theta\right)=\sqrt{\frac{1}{2 H_{0} \pi}} \sin \theta+\epsilon^{2} O_{5}(1) .
\end{aligned}
$$

Now we can give the estimates of $\Sigma_{1}$ and $\Sigma_{2}$. 
Lemma 17. The following estimates hold true:

$$
\begin{aligned}
\Sigma_{1}\left(t_{0}, \rho_{0}, 2 \pi ; \epsilon\right)= & \left(\frac{\pi}{2 \rho_{0}}\right)^{(\alpha-1) / 2} \\
& \times \int_{0}^{\pi}(\sin \theta)^{1+\alpha} \bar{p}_{+}\left(t_{0}+\theta\right) d \theta+o_{6}(1), \\
\Sigma_{2}\left(t_{0}, \rho_{0}, 2 \pi ; \epsilon\right)= & -\pi^{(1-\alpha) / 2}\left(2 \rho_{0}\right)^{(\alpha+1) / 2} \\
& \times \int_{0}^{\pi}(\sin \theta)^{1+\alpha} \bar{p}_{+}^{\prime}\left(t_{0}+\theta\right) d \theta+o_{6}(1),
\end{aligned}
$$

for $\epsilon \rightarrow 0$.

Proof. Firstly we consider $\Sigma_{1}$. By Lemmas 11, 12, and 15 and (77), we have

$$
\begin{aligned}
\Sigma_{1}\left(t_{0}, \rho_{0}, 2 \pi ; \epsilon\right) \\
=\pi \epsilon^{\alpha-1} \int_{0}^{\pi} \frac{\partial x}{\partial H}\left(\epsilon^{-2} \rho, \theta\right) p\left(x\left(\epsilon^{-2} \rho, \theta\right), t\right) d \theta \\
+\epsilon^{\alpha-1} \int_{0}^{\pi} \frac{\partial R_{1}}{\partial H}\left(x\left(\epsilon^{-2} \rho, \theta\right), t\right) \\
+\frac{\partial I_{-}}{\partial H}\left(x\left(\epsilon^{-2} \rho, \theta\right), t\right) d \theta \\
=\pi \epsilon^{\alpha-1} \int_{0}^{\pi} \frac{\partial x}{\partial H}\left(\epsilon^{-2} \rho_{0}, \theta\right) p\left(x\left(\epsilon^{-2} \rho_{0}, \theta\right), t_{0}+\theta\right) d \theta \\
+\epsilon^{\alpha} O_{6}(1) \\
=\pi \epsilon^{\alpha-1} \int_{\Theta_{+}} \frac{\partial x}{\partial H}\left(\epsilon^{-2} \rho_{0}, \theta\right) p\left(x\left(\epsilon^{-2} \rho_{0}, \theta\right), t_{0}+\theta\right) d \theta \\
+\pi \epsilon^{\alpha-1} \int_{\Theta_{-}} \frac{\partial x}{\partial H}\left(\epsilon^{-2} \rho_{0}, \theta\right) p\left(x\left(\epsilon^{-2} \rho_{0}, \theta\right), t_{0}+\theta\right) d \theta \\
+\epsilon^{\alpha} O_{6}(1) .
\end{aligned}
$$

By result (2) of Lemma 9, as $\epsilon \rightarrow 0$ which means $x \rightarrow$ $\infty$, we have

$$
\begin{aligned}
\pi \epsilon^{\alpha-1} & \int_{\Theta_{+}} \frac{\partial x}{\partial H}\left(\epsilon^{-2} \rho_{0}, \theta\right) p\left(x\left(\epsilon^{-2} \rho_{0}, \theta\right), t_{0}+\theta\right) d \theta \\
= & \pi \epsilon^{\alpha-1} \int_{\Theta_{+}} \frac{\partial x}{\partial H}\left(\theta, \epsilon^{-2} \rho\right)|x|^{\alpha} \bar{p}_{+}\left(t_{0}+\theta\right) d \theta \\
& +\epsilon^{\alpha} O_{6}(1) .
\end{aligned}
$$

By the measure of $\Theta_{-}$, we have

$$
\begin{aligned}
\pi \epsilon^{\alpha-1} \int_{\Theta_{-}} \frac{\partial x}{\partial H}\left(\epsilon^{-2} \rho_{0}, \theta\right) \\
\quad \times p\left(x\left(\epsilon^{-2} \rho_{0}, \theta\right), t_{0}+\theta\right) d \theta=\epsilon^{\alpha} O_{6}(1) .
\end{aligned}
$$

By (90) and (91), we have

$$
\begin{aligned}
\Sigma_{1}\left(t_{0}, \rho_{0}, 2 \pi ; \epsilon\right) & \\
& =\pi \epsilon^{\alpha-1} \int_{\Theta_{+}} \frac{\partial x}{\partial H}\left(\theta, \epsilon^{-2} \rho\right)|x|^{\alpha} \bar{p}_{+}\left(t_{0}+\theta\right) d \theta+\epsilon^{\alpha} O_{6}(1) \\
& =\pi \epsilon^{\alpha-1} \int_{0}^{\pi} \frac{\partial x}{\partial H}\left(\theta, \epsilon^{-2} \rho\right)|x|^{\alpha} \bar{p}_{+}\left(t_{0}+\theta\right) d \theta+\epsilon^{\alpha} O_{6}(1) \\
& =\left(\frac{\pi}{2 \rho_{0}}\right)^{(1-\alpha) / 2} \int_{0}^{\pi}(\sin \theta)^{\alpha+1} \bar{p}_{+}\left(t_{0}+\theta\right) d \theta+o_{6}(1) .
\end{aligned}
$$

Now we consider $\Sigma_{2}$. By Lemmas 11,12 , and 15 and (77), we have

$$
\begin{aligned}
\Sigma_{2}\left(t_{0}, \rho_{0}, 2 \pi ; \epsilon\right) & \\
= & -\pi \epsilon^{\alpha+1} \int_{0}^{\pi} \frac{\partial P}{\partial t}\left(x\left(\theta, \epsilon^{-2} \rho\right), t\right) d \theta \\
& -\epsilon^{\alpha+1} \int_{0}^{\pi}\left[\frac{\partial R_{1}}{\partial t}\left(x\left(\theta, \epsilon^{-2} \rho\right), t\right)\right. \\
= & +\pi \epsilon^{\alpha+1} \int_{0}^{\pi} \frac{\partial P}{\partial t}\left(x\left(\theta, \epsilon^{-2} \rho_{0}\right), t_{0}+\theta\right) d \theta+\epsilon^{\alpha} O_{6}(1) \\
= & \left.-\pi \epsilon^{\alpha+1} \int_{\Theta_{+}} \frac{\partial P}{\partial t}\left(x\left(\theta, \epsilon^{-2} \rho\right), t\right)\right] d \theta \\
& -\pi \epsilon^{\alpha+1} \int_{\Theta_{-}} \frac{\partial P}{\partial t}\left(x\left(\theta, \epsilon^{-2} \rho_{0}\right), t_{0}+\theta\right) d \theta+\epsilon^{\alpha} O_{6}(1) .
\end{aligned}
$$

By result (2) of Lemma 9, as $\epsilon \rightarrow 0$, we have

$$
\begin{gathered}
-\pi \epsilon^{\alpha+1} \int_{\Theta_{+}} \frac{\partial P}{\partial t}\left(x\left(\theta, \epsilon^{-2} \rho_{0}\right), t_{0}+\theta\right) d \theta \\
=-\frac{\pi \epsilon^{\alpha+1}}{\alpha+1} \int_{\Theta_{+}}\left|x\left(\theta, \epsilon^{-2} \rho_{0}\right)\right|^{\alpha} x\left(\theta, \epsilon^{-2} \rho_{0}\right) \bar{p}_{+}^{\prime}\left(t_{0}+\theta\right) d \theta \\
+\epsilon^{\alpha} O_{6}(1) .
\end{gathered}
$$

By the measure of $\Theta_{-}$, we have

$-\pi \epsilon^{\alpha+1} \int_{\Theta_{-}} \frac{\partial P}{\partial t}\left(x\left(\theta, \epsilon^{-2} \rho_{0}\right), t_{0}+\theta\right) d \theta=\epsilon^{\alpha} O_{6}(1)$. 
By (94) and (95), we have

$$
\begin{aligned}
\Sigma_{2}=-\frac{\pi \epsilon^{\alpha+1}}{\alpha+1} \int_{\Theta_{+}}\left|x\left(\theta, \epsilon^{-2} \rho_{0}\right)\right|^{\alpha} x\left(\theta, \epsilon^{-2} \rho_{0}\right) \bar{p}_{+}^{\prime} \\
\quad \times\left(t_{0}+\theta\right) d \theta+\epsilon^{\alpha} O_{6}(1) \\
=-\frac{\pi \epsilon^{\alpha+1}}{\alpha+1} \int_{0}^{\pi}\left|x\left(\theta, \epsilon^{-2} \rho_{0}\right)\right|^{\alpha} x\left(\theta, \epsilon^{-2} \rho_{0}\right) \bar{p}_{+}^{\prime} \\
\quad \times\left(t_{0}+\theta\right) d \theta+\epsilon^{\alpha} O_{6}(1) \\
=-\frac{1}{\alpha+1} \pi^{(1-\alpha) / 2}\left(2 \rho_{0}\right)^{(\alpha+1) / 2} \int_{0}^{\pi}(\sin \theta)^{1+\alpha} \bar{p}_{+}^{\prime} \\
\quad \times\left(t_{0}+\theta\right) d \theta+o_{6}(1) .
\end{aligned}
$$

Thus Lemma 17 is proved.

\subsection{Proof of Theorem 1. Let}

$$
\begin{aligned}
\Psi_{1}\left(t_{0}, \rho_{0}\right)= & \left(\frac{\pi}{2 \rho_{0}}\right)^{(1-\alpha) / 2} \\
& \times \int_{0}^{\pi}(\sin \theta)^{1+\alpha} \bar{p}_{+}\left(t_{0}+\theta\right) d \theta, \\
\Psi_{2}\left(t_{0}, \rho_{0}\right)= & -\frac{1}{\alpha+1} \pi^{(1-\alpha) / 2}\left(2 \rho_{0}\right)^{(\alpha+1) / 2} \\
& \times \int_{0}^{\pi}(\sin \theta)^{1+\alpha} \bar{p}_{+}^{\prime}\left(t_{0}+\theta\right) d \theta .
\end{aligned}
$$

Then there are two functions $\phi_{1}$ and $\phi_{2}$ such that the Poincaré map of (64), given by (76) of the form

$$
\begin{aligned}
& P: t_{1}=t_{0}+2 \pi+\epsilon^{1-\alpha} \Psi_{1}\left(t_{0}, \rho_{0}\right)+\epsilon^{1-\alpha} \phi_{1}, \\
& \rho_{1}=\rho_{0}+\epsilon^{1-\alpha} \Psi_{2}\left(t_{0}, \rho_{0}\right)+\epsilon^{1-\alpha} \phi_{2},
\end{aligned}
$$

where $\phi_{1}, \phi_{2} \in O_{6}(1)$.

Since $\int_{0}^{\pi} \bar{p}_{+}\left(t_{0}+\theta\right) \sin \theta d \theta>0, \forall t_{0} \in R$, we have

$$
\Psi_{1}>0, \quad \frac{\partial \Psi_{1}}{\partial \rho_{0}} \neq 0 .
$$

Let

$$
L=\frac{\rho_{0}^{-(1+\alpha) / 2}}{\int_{0}^{\pi}(\sin \theta)^{1+\alpha} \bar{p}_{+}\left(t_{0}+\theta\right) d \theta} .
$$

Then

$$
\frac{\partial L}{\partial t_{0}} \Psi_{1}\left(t_{0}, \rho_{0}\right)+\frac{\partial L}{\partial \rho_{0}} \Psi_{2}\left(t_{0}, \rho_{0}\right)=0
$$

The other assumptions of Ortega's theorem are easily verified. Hence, there is an invariant curve of $P$ in the annulus $\left(t_{0}, \rho_{0}\right) \in S^{1} \times[1.2]$ which implies the boundedness of our original equation (4). Then Theorem 1 is proved.
2.5. Proof of Theorem 2. We apply Aubry-Mather theory. By Theorem B in [10] and the monotone twist property of the Pioncaré map $P$ guaranteed by $\partial \Psi_{1} / \partial \rho_{0}<0$, it is straightforward to check that Theorem 2 is correct.

\section{References}

[1] M. Levi, "Quasiperiodic motions in superquadratic timeperiodic potentials," Communications in Mathematical Physics, vol. 143, no. 1, pp. 43-83, 1991.

[2] T. Küpper and J. You, "Existence of quasiperiodic solutions and Littlewood's boundedness problem of Duffing equations with subquadratic potentials," Nonlinear Analysis. Theory, Methods \& Applications A, vol. 35, pp. 549-559, 1999.

[3] X. Li, "Boundedness of solutions for Duffing's equations with semilinear potentials," Journal of Differential Equations, vol. 176, no. 1, pp. 248-268, 2001.

[4] Y. Wang, "Boundedness for sublinear Duffing equations with time-dependent potentials," Journal of Differential Equations, vol. 247, no. 1, pp. 104-118, 2009.

[5] A. Capietto, W. Dambrosio, and B. Liu, "On the boundedness of solutions to a nonlinear singular oscillator," Zeitschrift für Angewandte Mathematik und Physik, vol. 60, no. 6, pp. 10071034, 2009.

[6] B. Liu, "Quasi-periodic solutions of forced isochronous oscillators at resonance," Journal of Differential Equations, vol. 246, no. 9, pp. 3471-3495, 2009.

[7] R. Ortega, "Boundedness in a piecewise linear oscillator and a variant of the small twist theorem," Proceedings of the London Mathematical Society, vol. 79, no. 2, pp. 381-413, 1999.

[8] S. Jiang and F. Fang, "Lagrangian stability of a class of secondorder periodic systems," Abstract and Applied Analysis, vol. 2011, Article ID 106214, 28 pages, 2011.

[9] B. Liu, "Boundedness of solutions for equations with $p$ Laplacian and an asymmetric nonlinear term," Journal of Differential Equations, vol. 207, no. 1, pp. 73-92, 2004.

[10] M. L. Pei, "Aubry-Mather sets for finite-twist maps of a cylinder and semilinear Duffing equations," Journal of Differential Equations, vol. 113, no. 1, pp. 106-127, 1994. 


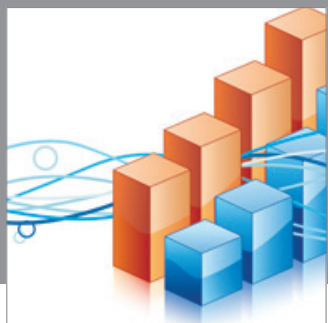

Advances in

Operations Research

mansans

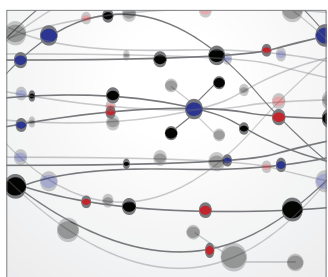

The Scientific World Journal
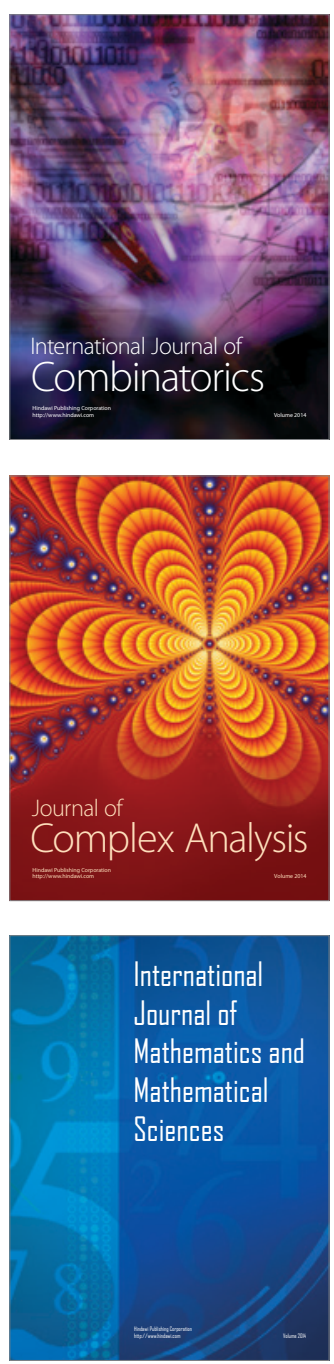
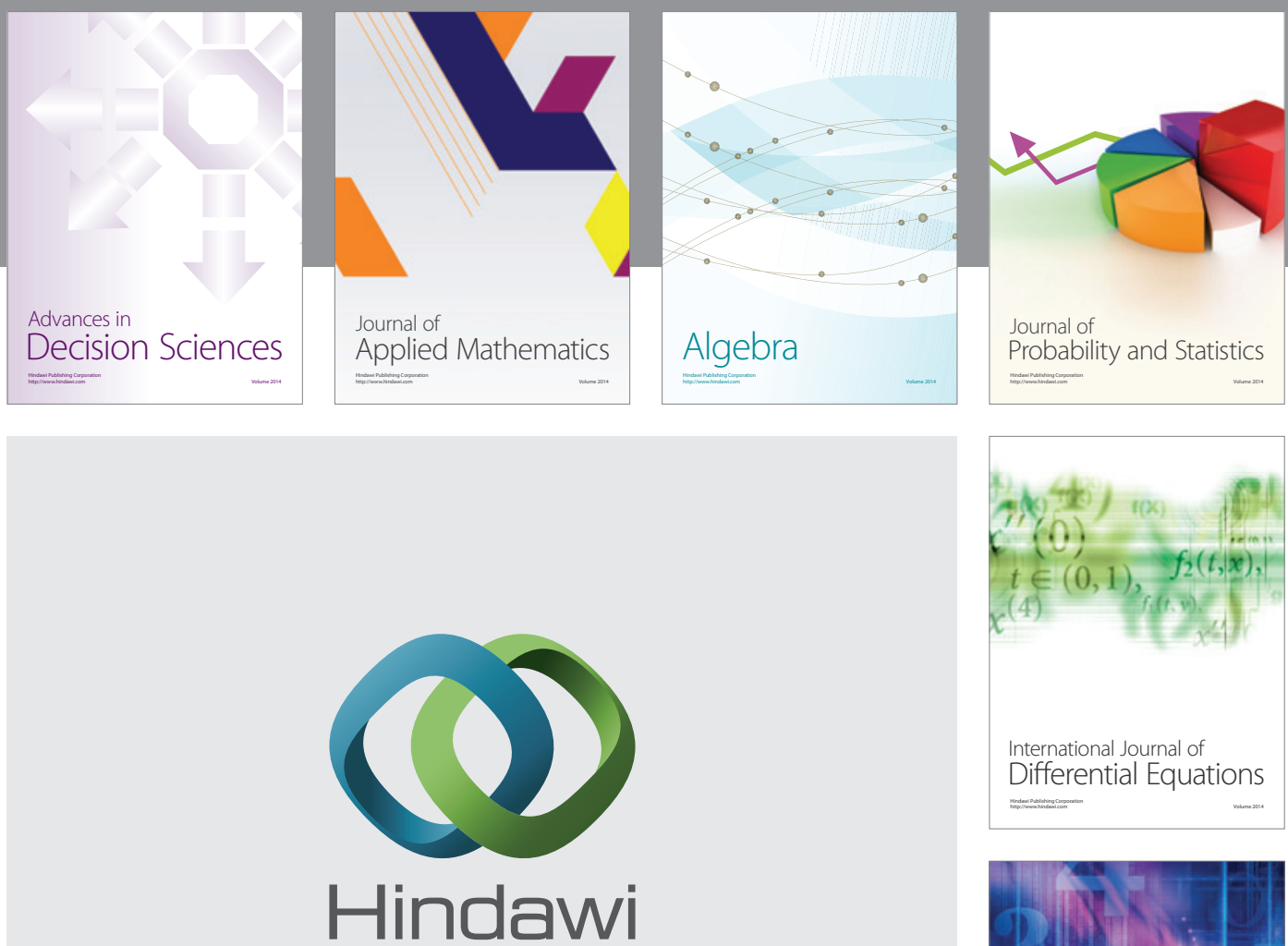

Submit your manuscripts at http://www.hindawi.com
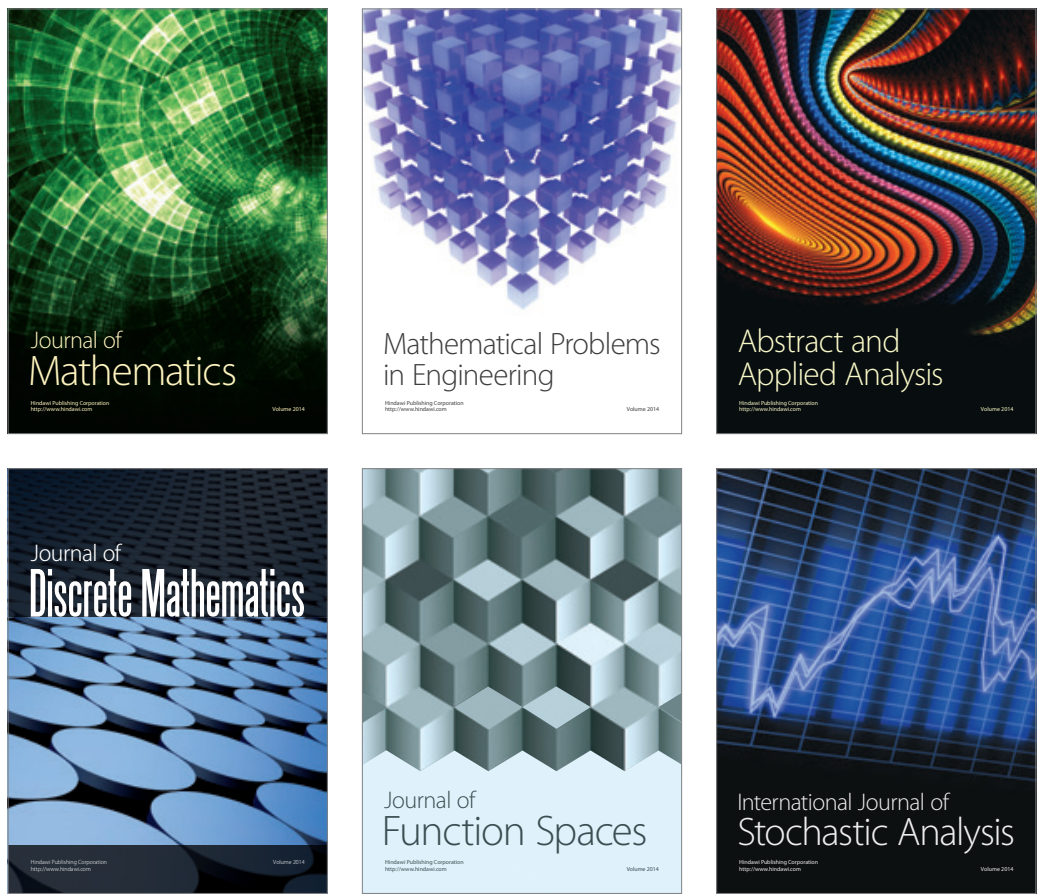

Journal of

Function Spaces

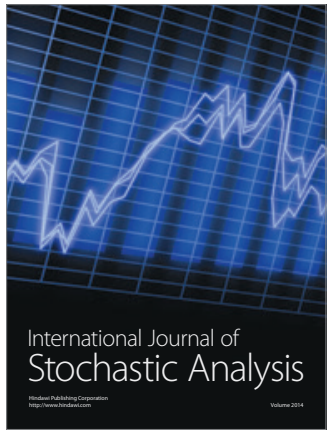

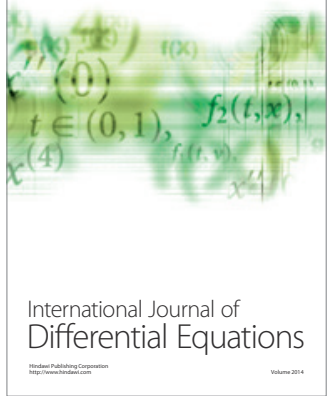
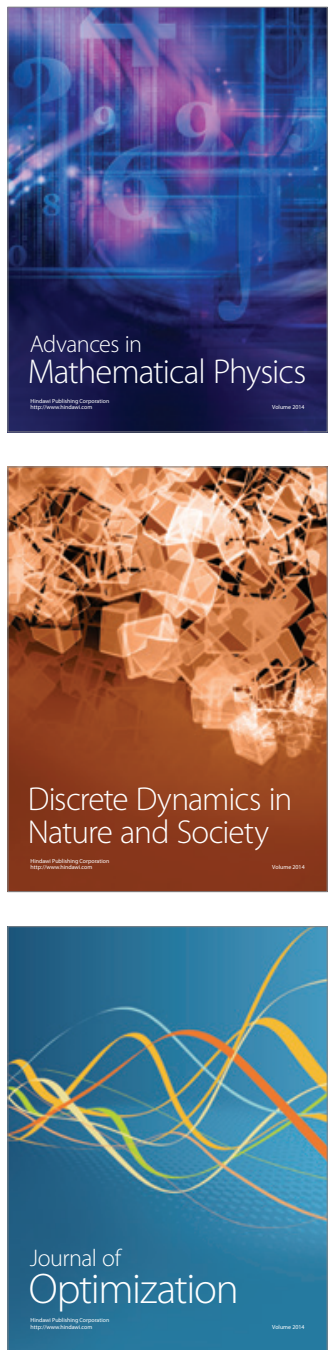